\title{
Determination of the Coverage of Phosphatidylcholine Monolayers Formed at Silicone Oil-Water Interfaces by Vesicle Fusion
}

\author{
Chiho Kataoka-Hamai* and Kohsaku Kawakami \\ Research Center for Functional Materials, National Institute for Materials Science \\ 1-1 Namiki, Tsukuba, Ibaraki 305-0044, Japan \\ *E-mail: kataoka.chiho@nims.go.jp
}

Number of pages: 5

Number of figures: 1

\section{SUPPORTING INFORMATION - \\ Energy Between a Vesicle and a Silicone Oil Surface}

Vesicle Surface Potential. The surface potential $(\psi)$ and surface charge density $(\sigma)$ have the following relation: ${ }^{1}$

$$
\sigma^{2}=2 \varepsilon_{\mathrm{r}} \varepsilon_{0} k T\left(\sum_{\mathrm{i}} \rho_{\mathrm{i}} \exp \left(-\frac{e z_{\mathrm{i}} \psi}{k T}\right)-\sum_{\mathrm{i}} \rho_{\mathrm{i}}\right)
$$

where $\varepsilon_{\mathrm{r}}$ is the relative permittivity of water (80.2), $\varepsilon_{0}$ is the permittivity of vacuum, $k$ is the Boltzmann constant, $T$ is the temperature, $e$ is the elementary charge, $\rho_{\mathrm{i}}$ is the number density of the ionic species in the bulk, and $z_{\mathrm{i}}$ is the ion valence. Thus, the vesicle surface potential $\left(\psi_{\mathrm{s}}\right)$ in buffer containing $10 \mathrm{mM}$ sodium phosphate ( $\mathrm{pH} 7.4$ ) and $\mathrm{NaCl}$ has the following relation with $\sigma:^{2}$

$$
\begin{gathered}
\sigma^{2}=2 \varepsilon_{\mathrm{r}} \varepsilon_{0} k T\left\{\rho_{\mathrm{Na}^{+}} \cdot \exp \left(-\frac{e \psi_{\mathrm{s}}}{k T}\right)+\rho_{\mathrm{Cl}^{-}} \cdot \exp \left(\frac{e \psi_{\mathrm{s}}}{k T}\right)+\rho_{\mathrm{H}_{2} \mathrm{PO}_{4}^{-}} \cdot \exp \left(\frac{e \psi_{\mathrm{s}}}{k T}\right)\right. \\
\left.+\rho_{\mathrm{HPO}_{4}^{2-}} \cdot \exp \left(\frac{2 e \psi_{\mathrm{s}}}{k T}\right)-\rho_{\mathrm{Na}^{+}}-\rho_{\mathrm{Cl}^{-}}-\rho_{\mathrm{H}_{2} \mathrm{PO}_{4}^{-}}-\rho_{\mathrm{HPO}_{4}^{2-}}\right\}
\end{gathered}
$$

$\sigma$ of POPC/TR-DHPE (99.5:0.5) and POPC/POPG (9:1) vesicles was estimated to be -0.0011 and $-0.023 \mathrm{C} / \mathrm{m}^{2}$, respectively, by assuming an area per lipid of $70 \AA^{2}{ }^{3}$ Using eq. $\mathrm{S} 2$, $\psi_{\mathrm{s}}$ of the POPC/TR-DHPE (99.5:0.5) vesicle was calculated to be $-3.0,-2.3,-1.9,-1.7$, and $-1.5 \mathrm{mV}$ at $\mathrm{NaCl}$ concentrations of $0,20,40,60$, and $80 \mathrm{mM}$, respectively. $\psi_{\mathrm{s}}$ of the POPC/POPG (9:1) vesicle was estimated to be $-57,-43,-37,-32$, and $-29 \mathrm{mV}$ at $\mathrm{NaCl}$ concentrations of $0,20,40,60$, and $80 \mathrm{mM}$, 
respectively. These $\psi_{\mathrm{s}}$ values are similar to those previously determined for phosphatidylcholine vesicles containing a negatively charged lipid. ${ }^{4}$

Oil Surface Potential. The $\zeta$-potential of silicone oil in $10 \mathrm{mM}$ sodium phosphate $(\mathrm{pH} 7.2)$ has been reported to be $-27 \mathrm{mV} .^{5}$ Thus, the surface potential of the oil surface $\left(\psi_{\mathrm{p}}\right)$ in $10 \mathrm{mM}$ sodium phosphate ( $\mathrm{pH} 7.2)$ was calculated by substituting this potential value in $\psi(x)$ in the following equation: ${ }^{1}$

$$
\psi(x)=\frac{2 k T}{e} \log \left[\frac{1+\gamma_{\mathrm{p}} \cdot \exp (-\kappa x)}{1-\gamma_{\mathrm{p}} \cdot \exp (-\kappa x)}\right]
$$

where $x$ is the distance from the surface. The inverse Debye screening length $(\kappa)$ and $\gamma_{\mathrm{p}}$ are given by

$$
\kappa=\sqrt{\frac{\sum_{\mathrm{i}} \rho_{\mathrm{i}} e^{2} z_{\mathrm{i}}^{2}}{\varepsilon_{\mathrm{r}} \varepsilon_{0} k T}}
$$

and

$$
\gamma_{\mathrm{p}}=\tanh \left(\frac{e \psi_{\mathrm{p}}}{4 k T}\right)
$$

respectively. Assuming that the shear plane was located $2 \mathrm{~nm}$ from the surface (i.e., $x=2 \mathrm{~nm}$ ), ${ }^{6,7}$ the $\psi_{\mathrm{p}}$ value in $10 \mathrm{mM}$ sodium phosphate $(\mathrm{pH} 7.2)$ was calculated to be $-101 \mathrm{mV}$. The $\sigma$ value was -0.058 $\mathrm{C} / \mathrm{m}^{2}$ according to eq. $\mathrm{S} 1$. The $\psi_{\mathrm{p}}$ values in the solutions used in the present study $(0-80 \mathrm{mM} \mathrm{NaCl}, 10$ $\mathrm{mM}$ sodium phosphate, $\mathrm{pH}$ 7.4) were obtained using eq. $\mathrm{S} 1$. The $\psi_{\mathrm{p}}$ values at $\mathrm{NaCl}$ concentrations of 0 , $20,40,60$, and $80 \mathrm{mM}$ were $-100,-83,-74,-67$, and $-62 \mathrm{mV}$, respectively.

Calculation of the Energy Between a Sphere and a Flat Plate. The interaction between the vesicle and the oil surface is represented as the interaction between a spherical particle (radius, $a$ ) and an infinite flat plate (Figure S1A, insert). The distance of closest approach of the two bodies is denoted $d$. The interaction energy was calculated according to the Derjaguin-Landau-Verwey-Overbeek (DLVO) theory. For simplicity, it was considered that the sphere and flat plate were immersed in a 1:1 electrolyte solution. In the experimental system, the ionic strength of the $\mathrm{NaCl}$-free buffer $(10 \mathrm{mM}$ sodium phosphate, $\mathrm{pH} 7.4$ ) was $28 \mathrm{mM}$. Therefore, in the calculation, the $\mathrm{NaCl}$-free buffer was approximated as the medium containing $28 \mathrm{mM}$ of the $1: 1$ electrolyte. The experimental value of the $\mathrm{NaCl}$ concentration was added to this offset. The total energy of the sphere-plate interaction $(U)$ is described by ${ }^{8}$

$$
U=U_{\mathrm{vdw}}+U_{\mathrm{el}}
$$

where $U_{\mathrm{vdw}}$ and $U_{\mathrm{el}}$ are the van der Waals and electrical double layer interactions, respectively. $U_{\mathrm{vdw}}$ is expressed as ${ }^{9}$

$$
U_{\mathrm{vdw}}=-\frac{H a}{6 d}\left(\frac{1}{1+14 d / \lambda}\right)
$$


where $H$ is the Hamaker constant and $\lambda$ is the characteristic wavelength. We assumed that $\lambda=100 \mathrm{~nm} .^{9}$ The Hamaker constant for the polydimethylsiloxane (PDMS)-water-PDMS interaction $\left(H_{\mathrm{pwp}}\right)$ has been reported to be $3.3 \times 10^{-22} \mathrm{~J} .{ }^{10}$ The Hamaker constant for the interaction between 1,2-dioleoyl-sn-glycero3-phosphocholine bilayers in water $\left(H_{\mathrm{bwb}}\right)$ has been reported to be $5.6 \times 10^{-21} \mathrm{~J}^{11}$ Thus, the Hamaker constant for the PDMS-water-bilayer interaction $\left(H_{\mathrm{pwb}}\right)$ was estimated to be $1.4 \times 10^{-21} \mathrm{~J}$ using the following expression: ${ }^{1}$

$$
H_{\mathrm{pwb}} \approx \sqrt{H_{\mathrm{pwp}} H_{\mathrm{bwb}}} .
$$

In 1:1 electrolyte solution, $U_{\mathrm{el}}$ is approximated as ${ }^{8,12}$

$$
U_{\mathrm{el}}=64 \pi a \varepsilon_{0} \varepsilon_{\mathrm{r}} \gamma_{\mathrm{s}} \gamma_{\mathrm{p}}\left(\frac{k T}{e}\right)^{2} \exp (-\kappa d)
$$

where $\gamma_{\mathrm{s}}$ is given by

$$
\gamma_{\mathrm{s}}=\tanh \left(\frac{e \psi_{\mathrm{s}}}{4 k T}\right) .
$$

Equation S9 is known to provide a good approximation for large particles $(\kappa a \geq 10) .{ }^{12}$ Our experimental systems were in this range. The total energy was calculated using the estimated values of $\psi_{\mathrm{s}}, \psi_{\mathrm{p}}$, and $H$ (Figure S1). 

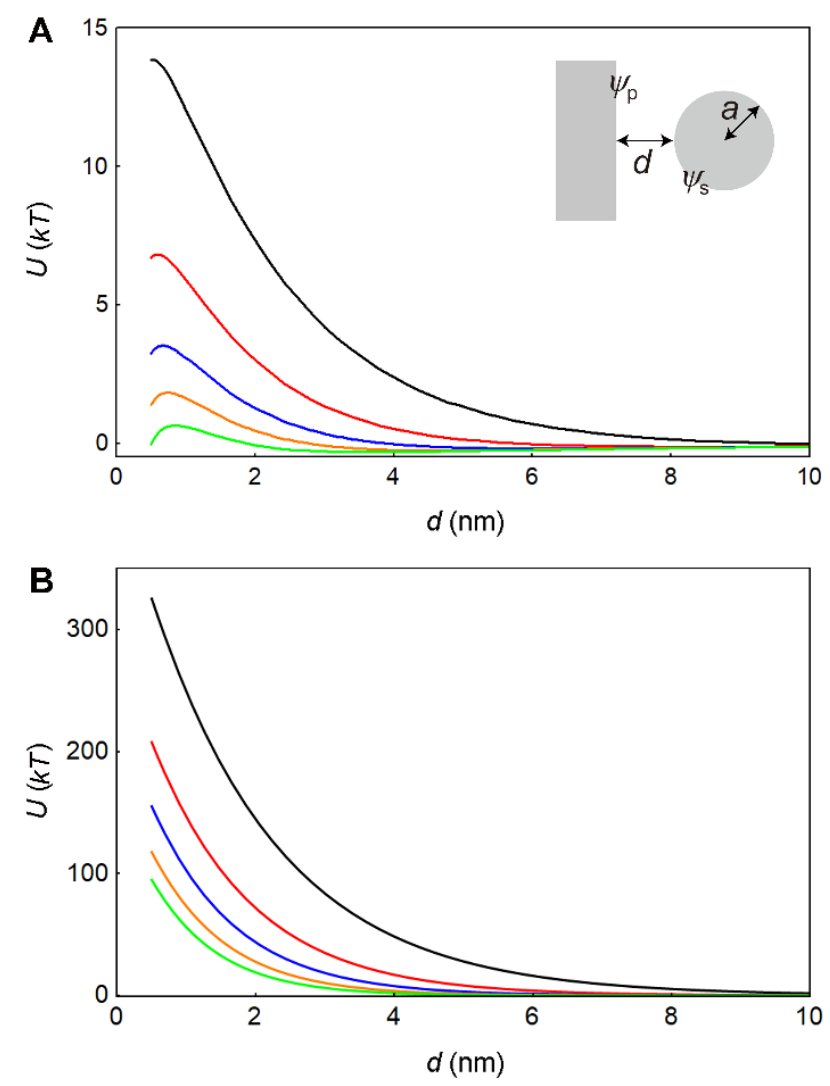

Figure S1. Energy $(U)$ between a spherical particle and a flat plate immersed in 1:1 electrolyte solution. The aqueous medium contained 28 (black), 48 (red), 68 (blue), 88 (orange), and $108 \mathrm{mM}$ (green) of the 1:1 electrolyte. These media correspond to the sodium phosphate buffer solutions containing 0 (black), 20 (red), 40 (blue), 60 (orange) and $80 \mathrm{mM} \mathrm{NaCl}$ (green) used in the experiments. In the insert in $\mathrm{A}, a, d, \psi_{\mathrm{s}}$, and $\psi_{\mathrm{p}}$ are the particle radius $(50 \mathrm{~nm})$, distance of closest approach, surface potential of the sphere, and surface potential of the plate, respectively. POPC/TR-DHPE (99.5:0.5) (A) and POPC/POPG (9:1) (B) vesicles.

\section{References}

1. Israelachvili, J. N., Intermolecular and Surface Forces, 2nd Edition. Academic Press: 1992.

2. Poyton, M. F.; Sendecki, A. M.; Cong, X.; Cremer, P. S., $\mathrm{Cu}^{2+}$ Binds to Phosphatidylethanolamine and Increases Oxidation in Lipid Membranes. J. Am. Chem. Soc. 2016, 138, 1584-1590.

3. Kučerka, N.; Tristram-Nagle, S.; Nagle, J. F., Structure of Fully Hydrated Fluid Phase Lipid Bilayers with Monounsaturated Chains. J. Membrane Biol. 2005, 208, 193-202.

4. Woodle, M. C.; Collins, L. R.; Sponsler, E.; Kossovsky, N.; Papahadjopoulos, D.; Martin, F. J., Sterically Stabilized Liposomes - Reduction in Electrophoretic Mobility but Not Electrostatic Surface Potential. Biophys. J. 1992, 61, 902-910.

5. Basu, P.; Blake-Haskins, A. W.; O'Berry, K. B.; Randolph, T. W.; Carpenter, J. F., Albinterferon $\alpha_{2 b}$ Adsorption to Silicone Oil-Water Interfaces: Effects on Protein Conformation, Aggregation, and Subvisible Particle Formation. J. Pharm. Sci. 2014, 103, 427-436.

6. Harding, I. H.; Healy, T. W., Electrical Double Layer Properties of Amphoteric Polymer Latex Colloids. J. Colloid Interface Sci. 1985, 107, 382-397.

7. Park, S. W.; Huang, C. P., The Surface Acidity of Hydrous CdS(s). J. Colloid Interface Sci. 1987, 
$117,431-441$.

8. Petosa, A. R.; Jaisi, D. P.; Quevedo, I. R.; Elimelech, M.; Tufenkji, N., Aggregation and Deposition of Engineered Nanomaterials in Aquatic Environments: Role of Physicochemical Interactions. Environ. Sci. Technol. 2010, 44, 6532-6549.

9. Gregory, J., Approximate Expressions for Retarded van der Waals Interaction. J. Colloid Interface Sci. 1981, 83, 138-145.

10. Koh, A.; Gillies, G.; Gore, J.; Saunders, B. R., Flocculation and Coalescence of Oil-in-Water Poly(dimethylsiloxane) Emulsions. J. Colloid Interface Sci. 2000, 227, 390-397.

11. Lis, L. J.; Mcalister, M.; Fuller, N.; Rand, R. P.; Parsegian, V. A., Interactions Between Neutral Phospholipid Bilayer Membranes. Biophys. J. 1982, 37, 657-665.

12. Bhattacharjee, S.; Elimelech, M., Surface Element Integration: A Novel Technique for Evaluation of DLVO Interaction Between a Particle and a Flat Plate. J. Colloid Interface Sci. 1997, 193, 273-285. 